ISSN 0103-5150

Fisioter. Mov., Curitiba, v. 24, n. 1, p. 157-166, jan./mar. 2011 Licenciado sob uma Licença Creative Commons

\title{
Diferenças biomecânicas entre os gêneros e sua importância nas lesões do joelho
}

\author{
Biomechanics differences between genders and its role in the \\ knee injuries
}

\section{Rodrigo de Marche Baldon ${ }^{[a]}$, Daniel Ferreira Moreira Lobato ${ }^{[b]}$, Lívia Pinheiro Carvalho ${ }^{[c]}$, Paloma Yan Lam Wun ${ }^{[\mathrm{d}]}$, Fábio Viadanna Serrão ${ }^{[\mathrm{e}]}$}

[a] Doutorando em Fisioterapia pela Universidade Federal de São Carlos (UFSCar), São Carlos, SP - Brasil, e-mail: rodrigo_baldon@hotmail.com

[b] Doutorando em Fisioterapia pela Universidade Federal de São Carlos (UFSCar), São Carlos, SP - Brasil, e-mail: daniellobato@ig.com.br

[c] Bolsista de iniciação científica da Fundação de Amparo à Pesquisa do Estado de São Paulo (FAPESP), no curso de Fisioterapia na Universidade Federal de São Carlos (UFSCar), São Carlos, SP - Brasil, e-mail: livia.pinheiro.carvalho@gmail.com

[d] Bolsista de iniciação científica do Conselho Nacional de Desenvolvimento Científico e Tecnológico (CNPq), no curso de Fisioterapia na Universidade Federal de São Carlos (UFSCar), São Carlos, SP - Brasil, e-mail: pylw_06@hotmail.com

[e] Doutor em Fisioterapia pela Universidade Federal de São Carlos (UFSCar), professor adjunto do Departamento de Fisioterapia da Universidade Federal de São Carlos (UFSCar), São Carlos, SP - Brasil, e-mail: fserrao@ufscar.br

\section{Resumo}

Introdução: Diversas pesquisas têm sido realizadas para determinar os fatores biomecânicos responsáveis pela maior incidência da síndrome da dor femoropatelar e das lesões do ligamento cruzado anterior em mulheres quando comparadas aos homens. Objetivo: 0 objetivo deste estudo foi realizar uma revisão de literatura não sistemática das diferenças biomecânicas entre os gêneros, especialmente aquelas referentes à articulação do quadril, potencialmente responsáveis pela maior incidência de lesões no joelho em mulheres, bem como verificar as suas implicações na elaboração de intervenções preventivas. Metodologia: Artigos publicados entre 1985 e 2008 foram selecionados por dois revisores independentes nas seguintes bases de dados: LILACS, MEDLINE, Cochrane e PubMed. Foram selecionados estudos teóricos, transversais e de correlação, assim como ensaios clínicos controlados, com desenhos experimentais bem esclarecidos. Resultados e discussão: Os estudos selecionados revelaram que as mulheres realizam atividades 
funcionais com menores ângulos de flexão de joelho e quadril, assim como maior valgo dinâmico do joelho, adução e rotação medial do quadril. Sugere-se, ainda, que as diferenças observadas nos planos coronal e transversal do quadril são mais significativas para a instalação de lesões nas mulheres do que aquelas que ocorrem unicamente no plano sagital. Por fim, as pesquisas demonstram que o treino neuromuscular melhora o alinhamento dinâmico do membro inferior nas mulheres, diminuindo a incidência de lesões no joelho. Conclusão: Há evidências de que as mulheres apresentam diferenças biomecânicas do padrão de movimento do membro inferior, quando comparadas aos homens, que podem contribuir para as maiores incidências de lesões no joelho observadas nesse gênero.

Palavras-chave: Ligamento cruzado anterior. Síndrome da dor femoropatelar. Quadril. Joelho. Fatores sexuais.

\section{Abstract}

Introduction: Several studies have been carried out to determine the biomechanical factors responsible by the greater incidence of patellofemoral pain syndrome and anterior cruciate ligament injury in women, when compared to men. Objective: The aim of this study was to perform a non-systematic review of the literature of the biomechanical differences between genders, especially those concerning to the hip joint, related to the higher incidence of knee injuries in women, and verify its implications for developing preventive strategies. Methodology: Articles published between 1985 and 2008 were selected by two independent reviewers of the follow data bases: LILACS, MEDLINE, Cocharane and PubMed. It was selected theoretical, transversal, and correlational studies as well as controlled clinical trial with experimental designs well defined. Results and discussion: The studies selected showed that women perform functional activities with lower angles of knee and hip flexion, as well as greater knee valgus, hip adduction and medial rotation. Furthermore, it is believed that the differences shown in the coronal and transverse planes of the hip are more significant for the development of injuries in women than those occurred only in the sagittal plane. Finally, the studies show that neuromuscular training carried out preventively improves the dynamic alignment of the lower limb in women, reducing the incidence of knee injuries on them. Conclusion: There are evidences that women have biomechanics differences of the lower limb movement pattern when compared to men that may contribute for the greater incidence of knee injuries observed in women.

Keywords: Anterior cruciate ligament. Patellofemoral pain syndrome. Hip. Knee. Sex factors.

\section{Introdução}

A maioria das lesões observadas no cenário esportivo acomete a articulação do joelho, e as mulheres apresentam maior incidência para muitas dessas lesões (1). Dentre elas, a síndrome da dor femoropatelar (SDFP) e as rupturas do ligamento cruzado anterior (LCA) têm recebido atenção especial, uma vez que acarretam altos custos de tratamento e favorecem o desenvolvimento de doenças incapacitantes (2). Assim, com o intuito de reduzir a incidência dessas lesões nas mulheres e evitar os transtornos oriundos desse problema, muitas pesquisas têm sido realizadas para determinar os fatores responsáveis por essa disparidade de lesões entre os gêneros.
Os fatores predisponentes para as rupturas do LCA e o desenvolvimento da SDFP nas mulheres têm sido divididos em três grupos principais: anatômicos, hormonais e biomecânicos. Atualmente, a ênfase principal dos estudos concerne às diferenças biomecânicas existentes entre os gêneros, ou seja, aos diferentes padrões de ativação muscular e de movimento adotados durante um gesto motor. Tais aspectos são enfatizados por serem passíveis de intervenção fisioterapêutica, ao contrário das características anatômicas e das variações hormonais (3). Além disso, como a origem das dores femoropatelares e a maioria das rupturas do LCA são de origem atraumática, supõe-se que existam diferenças entre os gêneros nos movimentos realizados cotidianamente e na prática esportiva, favorecendo o elevado acometimento nas mulheres (4). 
Um possível fator etiológico atribuído à alta incidência dessas lesões nas mulheres refere-se ao comprometimento dos músculos do quadril, e pode ser explicado pela teoria da cadeia cinética fechada, a qual pressupõe ser necessário ter uma boa estabilidade dessa articulação para controlar os movimentos dos segmentos distais durante atividades de descarga de peso. Se a função de uma das articulações do membro inferior apresenta-se inadequada, acredita-se que lesões possam ocorrer em outras articulações e estruturas, particularmente naquelas localizadas distalmente a ela (5).

Atualmente, propõe-se que uma disfunção da musculatura glútea das mulheres promoveria desarranjos biomecânicos nas articulações do membro inferior (6). Tais estudos sugerem que o aumento do valgo dinâmico do joelho, o qual é definido pela adução e rotação medial do quadril, assim como abdução e rotação lateral do joelho (7), é influenciado diretamente pela incapacidade dessa musculatura em controlar tal movimento (8). Como o LCA é um restritor secundário da abdução do joelho (9), a partir da hipótese de aumento do estresse na articulação femoropatelar, quando há exacerbação desse movimento $(8,10)$, torna-se adequado relacionar a atuação insuficiente dos músculos do quadril com a gênese das rupturas do LCA (6) e da SDFP em mulheres $(8,10)$.

Além disso, acredita-se que as mulheres não utilizam corretamente os músculos do quadril para dissipar a energia oriunda dos gestos esportivos, adotando estratégias biomecânicas diferentes quando comparadas aos homens. Andrews e Axe (11) introduziram o conceito "ligamento-dominante", caracterizado pelo aumento do valgo do joelho nas mulheres em razão da incapacidade da musculatura do membro inferior em absorver adequadamente as forças produzidas, resultando em excessiva sobrecarga do LCA. Há, ainda, evidências de que as mulheres utilizam preferencialmente o músculo quadríceps para aumentar a estabilidade dinâmica do joelho. Tal estratégia, quando acompanhada da ação reduzida dos músculos posteriores da coxa e dos extensores do quadril, tenderia a aumentar as forças de anteriorização da tíbia e, consequentemente, o estresse sobre o LCA (12).

Dessa forma, essa revisão tem como objetivo principal apresentar os resultados de estudos que tentaram verificar diferenças biomecânicas do membro inferior - com ênfase no quadril - entre os gêneros, além de analisar como tais distinções poderiam contribuir para a elevada incidência de lesões no joelho das mulheres. Por fim, será discutido como o fisioterapeuta poderia atuar diante dessas desordens, com o intuito de prevenir futuras lesões no joelho nas mulheres praticantes de atividade física.

\section{Metodologia}

Foi realizada uma revisão de literatura não sistemática nas bases de dados LILACS, MEDLINE, Cochrane e PubMed. Foram utilizados artigos publicados entre 1985 e 2008. Os descritores de texto utilizados foram: anterior cruciate ligament, patellofemoral pain syndrome, biomechanics, electromyography, kinetics, physical therapy modalities, hip, knee, sex factors. Os critérios de seleção dos estudos incluídos nessa revisão foram os seguintes:

a) artigos escritos em língua inglesa ou portuguesa;

b) estudos teóricos que justificassem os objetivos do presente estudo;

c) estudos transversais comparando variáveis biomecânicas (cinemática e cinética) do membro inferior entre os gêneros;

d) estudos de correlação associando a capacidade de geração de torque dos músculos do quadril e a cinemática do membro inferior durante atividades funcionais;

e) ensaios clínicos controlados verificando o efeito de treinos preventivos neuromusculares sobre parâmetros biomecânicos do membro inferior.

Os critérios de exclusão foram os seguintes:

a) artigos em outros idiomas que não os selecionados;

b) artigos com deficiência na descrição metodológica, principalmente no que se refere à seleção e ao poder da amostra, assim como dos materiais utilizados. Dois revisores selecionaram os estudos independentemente, avaliaram sua qualidade metodológica, assim como a relevância dos resultados desses estudos para a presente revisão. Os artigos somente foram selecionados se ambos os revisores estivessem de acordo com a sua inclusão. 


\section{Resultados e discussão}

Diferenças biomecânicas do membro inferior entre os gêneros no plano sagital

Há evidências na literatura sugerindo que, durante a desaceleração de movimentos esportivos, as mulheres apresentam menores ângulos de flexão do joelho no contato inicial com o solo $(13,14)$ e nos valores máximos de amplitude de movimento (12, 15), além de possuírem ativação eletromiográfica aumentada do quadríceps $(12,16)$ e reduzida dos isquiotibiais (12) quando comparadas aos homens. Como a contração preferencial do quadríceps nos primeiros $30^{\circ}$ de flexão do joelho promove maior estresse sobre o LCA, assume-se que tal estratégia de movimento esteja relacionada com a maior incidência de lesões desse ligamento nas mulheres (12).

Aliado a esses achados, ao contrário dos homens, os quais utilizam a musculatura extensora do quadril para dissipar uma grande quantidade de energia produzida durante a fase de aterrissagem de um gesto esportivo, acredita-se que as mulheres possam adotar estratégias que requerem, preferencialmente, os músculos quadríceps e gastrocnêmio para essa finalidade (13). Zazulak et al. (3), ao avaliarem 22 atletas durante a aterrissagem unipodal, verificaram que as mulheres apresentaram maior ativação eletromiográfica do quadríceps e menor ativação do glúteo máximo quando comparadas aos homens. Assim, os autores relataram que o aumento da atividade do quadríceps combinada à redução da atividade do glúteo máximo poderia contribuir para alterar a absorção de energia durante a aterrissagem, sobrecarregando o LCA.

McLean et al. (14) realizaram uma avaliação cinemática com jogadores de basquetebol durante três atividades envolvendo mudança brusca de direção e concluíram que as mulheres apresentavam, durante a manobra do shuttle-run, menor ângulo de flexão do quadril no início da fase de apoio e menor pico de flexão do quadril. Similarmente, McLean et al. (17) e Salci et al. (18) relataram que as mulheres possuíam menor pico de flexão de quadril durante a fase de apoio em atividades de mudança de direção e no bloqueio realizado no voleibol, respectivamente. Dessa forma, tais autores acreditam que esse comportamento, associado ao fato das mulheres apresentarem menores ângulos de flexão do joelho e aumento das forças de reação do solo, predisporia a uma situação favorável à ruptura do LCA, uma vez que a musculatura extensora do quadril não estaria contribuindo de modo adequado para a dissipação de energia.

Ao contrário dos estudos acima citados, os quais realizaram uma avaliação durante o período de contato do membro inferior com o solo, Chappell et al. (16) avaliaram atletas recreacionais durante a fase aérea do vertical stop-jump (movimento realizado frequentemente por jogadores de basquetebol previamente ao arremesso) e verificaram que as mulheres exibiram menores ângulos de flexão do quadril antes da aterrissagem. Assim, os autores acreditam que há um padrão de movimento diferenciado nas mulheres desde a preparação da aterrissagem, o qual deveria ser enfocado durante as intervenções preventivas de lesões no joelho.

Na mesma tendência, Decker et al. (13), ao compararem variáveis cinemáticas, cinéticas e de absorção de energia (trabalho) entre os gêneros durante a aterrissagem de uma plataforma elevada, verificaram que as mulheres apresentaram menor ângulo de flexão do joelho e maior flexão plantar do tornozelo no contato inicial com o solo. Embora o ângulo de flexão do quadril no contato inicial - assim como o pico de flexão do quadril - não tenha sido diferente entre os gêneros, as mulheres apresentaram maior absorção de energia por meio do joelho e do tornozelo, quando comparados ao quadril, enquanto os homens não demonstraram diferenças entre as articulações. Dessa forma, os autores concluem que as mulheres, aterrissando em uma posição mais ereta, são incapazes de dissipar a energia cinética produzida durante a aterrissagem de modo equilibrado entre as articulações do membro inferior, resultando em uma possível sobrecarga da articulação do joelho.

Embora esses estudos tenham verificado diferenças cinemáticas e de ativação neuromuscular do quadril entre os gêneros no plano sagital, outras pesquisas não identificaram tais alterações durante a aterrissagem de uma plataforma (15), corrida (4) e salto unipodal horizontal $(6,19)$. Além disso, McLean et al. (20) verificaram que os momentos em extensão do joelho e as forças isoladas de cisalhamento anterior sobre a tíbia são incapazes de romper o LCA durante uma atividade de mudança abrupta de direção. Esse estudo, o qual utilizou uma técnica validada recentemente (21), consistiu em uma simulação computadorizada, baseada na 
análise cinemática do movimento, capaz de predizer o efeito de perturbações do sistema neuromuscular sobre os movimentos e sobrecargas da articulação do joelho. Dessa forma, sugere-se que as alterações do quadril no plano sagital, quando consideradas isoladamente, sejam incapazes de promover uma sobrecarga no joelho suficiente para a ruptura do LCA, tornando improvável que o padrão de movimento diferenciado adotado pelas mulheres nesse plano seja responsável, por si só, pela disparidade de lesões no joelho observada entre os gêneros.

Diferenças biomecânicas do membro inferior entre os gêneros nos planos frontal e transversal

Para melhor compreender como um movimento alterado do quadril nos planos frontal e transversal em uma atividade em cadeia cinética fechada predisporia à SDFP e às rupturas do LCA, é necessário realizar uma breve revisão de conceitos anatômicos e biomecânicos das articulações femoropatelar e femorotibial, respectivamente.

Classicamente, o ângulo do quadríceps - ou ângulo $\mathrm{Q}$ - tem sido estudado nas disfunções femoropatelares por causa da sua importância na geração de estresse nessa articulação. 0 ângulo $Q$, formado pela interação resultante não colinear de duas forças primárias que agem sobre a patela no plano frontal - o vetor de força do quadríceps $\left(\mathrm{F}_{\mathrm{Q}}\right)$ e o vetor de força do tendão patelar -, possui sua resultante de força dirigida lateralmente, podendo ser influenciada diretamente por qualquer mudança que ocorra na obliquidade desses dois vetores. Assim, uma maior obliquidade de $\mathrm{F}_{\mathrm{Q}}$ ocasionada, por exemplo, pela adução e rotação medial do quadril aumentaria a resultante lateral sobre a patela. Tal situação ocasionaria compressão excessiva da faceta lateral da patela sobre a proeminência lateral da tróclea do fêmur (quando o joelho está em extensão) ou no aspecto lateral da fossa intercondilar do fêmur (quando o joelho está em flexão) (22), predispondo à SDFP.

Em relação às rupturas do LCA, torna-se importante o esclarecimento dos mecanismos que promovem a lesão desse ligamento e como os movimentos do quadril poderiam contribuir para isso. Assim, tais mecanismos têm sido divididos em traumáticos e atraumáticos, sendo o primeiro originado por uma força aplicada diretamente sobre o joelho, frequentemente associada a esportes de contato físico, enquanto as rupturas do LCA de caráter atraumático, as quais ocorrem em até $88 \%$ dos casos (23), surgem de situações em que não há contato físico direto, como durante a aterrissagem de um salto. Tais lesões podem ser classificadas ainda quanto à natureza do estresse sobre o ligamento, sendo divididas em lesões por sobrecarga direta ou por impacto do LCA (24). Markolf et al. (9) verificaram que os movimentos isolados de abdução e rotação medial do joelho são capazes de produzir sobrecarga direta sobre o LCA, não sendo a mesma situação aplicável à rotação lateral isolada dessa articulação. No entanto, Fung e Zhang (24) relataram que esse movimento, quando associado com a abdução do joelho, induz o impacto do LCA na parede lateral da fossa intercondilar do fêmur, aumentando o estresse sobre essa estrutura.

Recentemente, Powers (8) relatou que alterações biomecânicas na articulação do quadril poderiam atuar como possíveis fatores etiológicos da SDFP. Segundo esse autor, a rotação medial e a adução excessiva do quadril, durante atividades em cadeia cinética fechada, aumentariam o ângulo $Q$ e alterariam a trajetória patelar, predispondo o indivíduo ao desenvolvimento da dor na articulação femoropatelar. Ireland (7) descreve, ainda, que o membro inferior, na maioria das lesões atraumáticas do LCA, assume uma posição composta por adução e rotação medial do quadril, assim como abdução e rotação lateral do joelho (valgo dinâmico). Como o LCA é um restritor secundário do movimento de abdução da articulação do joelho (9) e esse movimento combinado à rotação lateral dessa articulação induz o impacto dessa estrutura contra a parede lateral da fossa intercondilar do fêmur (24), acredita-se que a falta de controle dos movimentos do quadril nos planos frontal e transversal contribuiria para a aquisição de posturas favoráveis à ruptura do LCA.

Hewett et al. (25), em um estudo prospectivo com atletas, verificaram que as mulheres com maiores ângulos de valgo dinâmico e momento em valgo do joelho apresentaram maior incidência de lesões do LCA durante a temporada. Ainda, como o valgo do joelho parece ser um movimento associado a tais lesões, inúmeras pesquisas $(4,12,14,26-29)$ foram realizadas com o intuito de verificar diferenças desse movimento entre os gêneros durante atividades que mimetizam gestos esportivos. Além disso, torna-se interessante iniciar a discussão a respeito 
da influência do quadril sobre a elevada incidência dessas lesões em mulheres analisando tal variável, uma vez que os movimentos do joelho e do quadril, no plano coronal, têm sido correlacionados (29).

Ford et al. (26), ao avaliarem jogadores de basquetebol, demonstraram que as mulheres apresentavam maior excursão e máximo ângulo em valgo do joelho na aterrissagem bipodal de uma plataforma elevada. Similarmente, Russell et al. (27) verificaram que as mulheres, durante a aterrissagem unipodal, possuíam maior ângulo em valgo do joelho no contato inicial com o solo e, embora ambos os gêneros tenham adotado um padrão em varo no ângulo máximo de flexão de joelho, as mulheres obtiveram menores valores para esse movimento. Outros trabalhos na literatura, avaliando diferentes gestos esportivos, como a corrida $(4,12)$, a caminhada (28) e a mudança de direção $(12,14,30)$, também relataram maior valgo dinâmico nas mulheres. Dessa forma, sugere-se que a diferença entre os gêneros nos ângulos em valgo do joelho durante os gestos esportivos, assim como nas posturas adotadas pelas mulheres previamente à aterrissagem no solo, é consequente a um alterado controle muscular do membro inferior e, provavelmente, reflete diferenças no padrão de contração dos músculos periarticulares do joelho, bem como dos músculos do quadril (29), contribuindo para a disparidade de lesões entre os gêneros.

Embora seja conhecido que o valgo dinâmico do joelho é composto tanto pela abdução e rotação lateral do joelho quanto pela adução e rotação medial do quadril (3), poucos estudos compararam os padrões de movimento do quadril entre os gêneros no plano frontal e transversal. Ferber et al. (4) verificaram que as mulheres, durante a fase de apoio da corrida, demonstraram maior adução e rotação medial do quadril, assim como rotação lateral e abdução do joelho. Além disso, apresentaram maior trabalho negativo do quadril no plano frontal e transversal, sugerindo que houve maior exigência excêntrica dos músculos abdutores e rotadores laterais do quadril para controlar tais movimentos. Hurd et al. (28) observaram que as mulheres apresentaram maior excursão do quadril nos planos frontal (adução) e transversal (rotação medial) durante a caminhada, enquanto Zeller et al. (31), durante o agachamento unipodal, observaram que as mulheres adotaram uma postura em adução do quadril, corroborando a hipótese de que os músculos abdutores e rotadores laterais dessa articulação são mais exigidos nas mulheres para controlar esses movimentos.

Outra atividade funcional estudada com o objetivo de verificar diferenças biomecânicas do quadril entre os gêneros é a aterrissagem, uma vez que se trata do principal mecanismo de ruptura do LCA (23). Ford et al. (29) relataram que, durante a realização desse movimento, além de apresentar maior ângulo em valgo do joelho no contato inicial com o solo e maior pico desse movimento na fase de amortecimento, as mulheres exibiram maior excursão do quadril no plano frontal e pico do ângulo de adução dessa articulação. De modo complementar, Lephart et al. (15) verificaram que durante a aterrissagem as mulheres apresentaram maiores ângulos em rotação medial do quadril quando comparadas aos homens, enquanto Chappell et al. (16), ao avaliarem a preparação da aterrissagem do vertical stop-jump, observaram que as mulheres apresentavam uma fase aérea com menor abdução e rotação lateral do quadril, sugerindo que as alterações biomecânicas entre os gêneros existem antes mesmo de haver o contato do pé com o solo.

Embora existam hipóteses sobre o papel dos músculos abdutores e rotadores laterais do quadril no controle do fêmur e, consequentemente, nos movimentos do joelho durante atividades em cadeia cinética fechada (8), poucos trabalhos estudaram a possível influência desses músculos sobre tais articulações em ambos os gêneros. Willson et al. (32) verificaram redução da força isométrica dos abdutores do quadril e aumento do valgo dinâmico do joelho nas mulheres durante o agachamento unipodal, porém, não verificaram uma correlação significativa entre essas variáveis. Por outro lado, Claiborne et al. (33) encontraram um aumento do valgo dinâmico do joelho nas mulheres, bem como uma correlação negativa entre o torque excêntrico abdutor do quadril e esse movimento. A divergência dos dados obtidos pelos autores pode ser justificada pelas diferenças metodológicas dos estudos: no último (33), os voluntários realizaram o agachamento até $60^{\circ} \mathrm{de}$ flexão do joelho e tiveram a função excêntrica dos músculos abdutores do quadril avaliada; enquanto no primeiro (32) o ângulo do joelho avaliado foi o de $45^{\circ}$ de flexão e a avaliação de força foi isométrica. Assim, como o componente excêntrico de contração desses músculos está intimamente relacionado com as características de controle do movimento do quadril no plano frontal durante o agachamento, 
principalmente em uma situação de maior demanda energética, assume-se que essas peculiaridades tenham refletido de forma mais apropriada a relação entre tais variáveis.

Jacobs e Mattacola (19) e Jacobs et al. (6) estudaram as diferenças de força dos músculos abdutores do quadril e da cinemática do joelho no plano frontal entre os gêneros durante a aterrissagem unipodal de um salto horizontal. No primeiro estudo, não houve diferença do pico de torque excêntrico abdutor do quadril entre os gêneros e as mulheres apresentaram tendência a ter maior pico do ângulo em abdução do joelho, enquanto no segundo trabalho verificou-se diferença do pico de torque isométrico dos abdutores do quadril entre os gêneros, assim como menor excursão em abdução do joelho nos homens. Além disso, ambos os trabalhos verificaram uma correlação negativa entre a força dos músculos abdutores do quadril com os valores de abdução do joelho.

Diante do exposto, são observadas evidências que sugerem diferenças entre gêneros com relação às estratégias biomecânicas do quadril durante a realização de um gesto motor. É importante ressaltar que tais distinções têm sido observadas nos três planos de movimento do quadril, embora as alterações nos planos frontal e transversal pareçam ser as principais responsáveis em influenciar a aquisição de posturas que favoreçam o elevado índice de lesões no joelho nas mulheres.

Treino neuromuscular com ênfase na musculatura lombo-pélvica e sua ação preventiva sobre as lesões no joelho

Muitos programas de prevenção de lesões têm sido desenvolvidos com o intuito de modificar algumas características neuromusculares e biomecânicas que potencialmente contribuiriam para o elevado índice de rupturas do LCA e desenvolvimento da SDFP nas mulheres atletas. Tradicionalmente, tais programas incorporam uma combinação de atividades que envolvem desde alongamento e fortalecimento muscular até os conceitos de pliometria e exercícios de agilidade. Embora tais programas tenham demonstrado eficiência para a redução do número de lesões do $\operatorname{LCA}(2,34)$, pouco se sabe a respeito dos efeitos biomecânicos de tais protocolos sobre o membro inferior. São escassos, ainda, os trabalhos que realizaram um fortalecimento específico da musculatura lombo-pélvica e que verificaram a influência deste no gesto motor das mulheres.

Hewett et al. (35) submeteram onze mulheres atletas a um treino pliométrico de seis semanas, o qual objetivava o aprimoramento do gesto de aterrissar, além de exercícios de alongamento e fortalecimento de músculos do tronco, do membro superior e do inferior. Após o treino, os autores verificaram que as mulheres aumentaram a razão de torque dos músculos isquiotibiais/quadríceps, reduziram em $22 \%$ o pico de força de reação do solo e minimizaram os momentos em adução e abdução do joelho. Noyes et al. (36) verificaram a influência desse mesmo treino sobre a distância entre os joelhos durante a aterrissagem de uma plataforma elevada. Os autores concluíram que, após o treino, as mulheres aterrissaram com maior afastamento dos joelhos, refletindo alteração do comportamento do membro inferior no plano coronal. Myer et al. (37) analisaram a eficiência de um treino neuromuscular de seis semanas (constituído por exercícios pliométricos, de equilíbrio, de fortalecimento dos membros superiores e inferiores e de estabilização lombo-pélvica) sobre a performance e variáveis cinemáticas e cinéticas do membro inferior, concluindo que o treino melhorou tanto a performance das atletas durantes os testes funcionais como reduziu os momentos em valgo e varo do joelho durante a aterrissagem.

Embora não tenham sido citados nesses estudos, os músculos abdutores e rotadores laterais do quadril podem ter contribuído para as alterações biomecânicas observadas após o treino neuromuscular nas mulheres. Mesmo não sendo enfocado no programa preventivo, o treino pliométrico enfatizando a aterrissagem com a manutenção do alinhamento do membro inferior nos planos coronal e transversal poderia modificar o trofismo e o padrão de recrutamento desses músculos, uma vez que estes agem excentricamente para controlar os movimentos do fêmur durante esse gesto esportivo. Myer et al. (38) compararam o efeito do treino pliométrico com o de estabilização lombo-pélvica sobre variáveis cinemáticas do membro inferior durante atividades que possuíam um componente de aterrissagem, relatando que ambos foram efetivos em reduzir os ângulos de adução do quadril e abdução do joelho no contato inicial com o solo, assim como os ângulos máximos dessas variáveis. Pollard et al. (39), após submeterem 18 jogadoras de futebol a 
um treino composto por alongamentos, fortalecimento, exercícios pliométricos e de agilidade, nos quais eram enfatizadas a técnica correta de aterrissagem e a mudança de direção do movimento, verificaram redução da rotação medial do quadril e aumento dos ângulos de abdução do quadril durante a aterrissagem. Apesar de não terem avaliado a força e a atividade eletromiográfica dos músculos abdutores e rotadores laterais do quadril, os autores acreditam que esses músculos influenciaram na melhora do padrão de movimento das mulheres após os treinos.

Na literatura consultada, foi encontrado apenas um estudo no qual os autores verificaram o efeito de um fortalecimento específico em cadeia cinética aberta dos músculos abdutores e rotadores laterais do quadril sobre variáveis cinemáticas e cinéticas do membro inferior em mulheres (40). Embora tenha havido aumento significativo de força desses músculos, não foram encontradas alterações das outras variáveis analisadas. Dessa forma, sugere-se que a realização isolada de exercícios de fortalecimento em cadeia cinética aberta, ainda que eficiente para o aumento de força muscular, não tenha proporcionado um estímulo suficiente para promover alterações das estratégias de recrutamento muscular que atuassem para a modificação da cinemática do membro inferior. É possível que a incorporação de atividades funcionais ao treino preventivo, enfocando a ativação dos músculos do quadril, promova mudanças do planejamento motor e, associadamente aos ganhos de força muscular, favoreça a realização segura do gesto esportivo pelas mulheres.

\section{Considerações finais}

Tem sido observado que a SDFP e as rupturas do LCA apresentam maior incidência nas mulheres atletas quando comparadas aos homens praticantes das mesmas modalidades esportivas. Embora a origem dessa diferença aparente ser multifatorial, acredita-se que, nas situações em que não há trauma associado à etiologia, tais lesões possam se manifestar graças a um alterado padrão de movimento do quadril adotado pelas mulheres. Existem evidências de que as mulheres apresentam diminuição dos ângulos de flexão do quadril e aumento dos ângulos de adução e rotação medial dessa articulação durante atividades esportivas. Tal postura, relacionada com a fraqueza dos músculos abdutores e rotadores laterais do quadril, poderia induzir um aumento do valgo dinâmico do joelho e, dessa forma, aumentar o estresse na articulação femoropatelar e sobre o LCA.

Embora alguns trabalhos demonstrem diferenças biomecânicas na articulação do quadril entre os gêneros, o número de estudos dedicados a este assunto ainda é incipiente, indicando a necessidade de novos trabalhos e avaliando diferentes manobras esportivas para verificar se os padrões de movimento feminino alterados são específicos a determinados gestos motores. Sugere-se, ainda, a realização de estudos longitudinais para determinar a influência dos movimentos e da função alterada dos músculos do quadril na elevada incidência de lesão no joelho em mulheres.

Quanto aos estudos que realizaram um treino neuromuscular, verificou-se diminuição da incidência de lesões do LCA no grupo de mulheres tratadas previamente às temporadas de competição e melhora do alinhamento dinâmico do membro inferior. No entanto, a maioria desses trabalhos não avaliou as medidas objetivas que poderiam ter contribuído para a redução das taxas de lesão e a melhora da cinemática do membro inferior, como, por exemplo, a força dos músculos do quadril. Além disso, como os treinos neuromusculares são muito abrangentes, seria interessante verificar qual componente do programa de prevenção estaria mais relacionado com a redução da incidência de lesões nas mulheres e, dessa forma, contribuir com a otimização da intervenção.

\section{Referências}

1. Taunton JE, Ryan MB, Clement DB, McKenzie DC, Lloyd-Smith DR, Zumbo DB. A retrospective casecontrol analysis of 2002 running injuries. Br J Sports Med. 2002;36(2):95-101.

2. Hewett TE, Lindenfeld TN, Riccobene JV, Noyes FR. The effect of neuromuscular training on the incidence of knee injury in female athletes: a prospective study. Am J Sports Med. 1999;27(6):699-706.

3. Zazulak BT, Ponce PL, Straub SJ, Medvecky MJ, Avedisian L, Hewett TE. Gender comparison of hip muscle activity during single-leg landing. J Orthop Sports Phys Ther. 2005;35(5):292-9. 
4. Ferber R, Davis IM, Williams DS. Gender differences in lower extremity mechanics during running. Clin Biomech. 2003;18(4):350-7.

5. Prentice WE, Voight MI. Techniques in musculoskeletal rehabilitation. New York: McGraw Hill; 2001.

6. Jacobs CA, Uhl T, Mattacola CG, Shapiro R, Rayens WS. Hip abductor function and lower extremity landing kinematics: sex differences. J Athl Train. 2007; 42(1):76-83.

7. Ireland ML. Anterior cruciate ligament injury in female athletes: epidemiology. J Athl Train. 1999;34(2): 150-4.

8. Powers CM. The influence of altered lower-extremity kinematics on patellofemoral joint dysfunction: a theoretical perspective. J Orthop Sports Phys Ther. 2003;33(11):639-46.

9. Markolf KL, Burchfield DM, Shapiro MM, Shepard MF, Finerman GA, Slauterbeck JL. Combined knee loading states that generate high anterior cruciate ligament forces. J Orthop Res. 1995;13(6):930-5.

10. Cichanowski HR, Schmitt JS, Johnson RJ, Niemuth PE. Hip strength in collegiate female athletes with patellofemoral pain. Med Sci Sports Exerc. 2007;39(8): 1227-32.

11. Andrews JR, Axe MJ. The classification of knee ligament instability. Orthop Clin North Am. 1985;16(1): 69-82.

12. Malinzak RA, Colby SM, Kirkendall DT, Yu B, Garrett WE. A comparison of knee joint motion patterns between men and women in selected athletic tasks. Clin Biomech. 2001;16(5):438-45.

13. Decker MJ, Torry MR, Wyland DJ, Sterett WI, Steadman JR. Gender differences in lower extremity kinematics, kinetics and energy absorption during landing. Clin Biomech. 2003;18(7):662-9.

14. McLean SG, Walker KB, van den Bogert AJ. Effect of gender on lower extremity kinematics during rapid direction changes: an integrated analysis of three sports movements. J Sci Med Sport. 2005;8(4): 411-22.

15. Lephart SM, Ferris CM, Riemann BL, Myers JB, Fu FH. Gender differences in strength and lower extremity kinematics during landing. Clin Orthop Relat Res. 2002;(401):162-9.
16. Chappell JD, Creighton RA, Giuliani C, Yu B, Garrett WE. Kinematics and electromyography of landing preparation in vertical stop-jump: risks for noncontact anterior cruciate ligament injury. Am J Sports Med. 2007;35(2):235-41.

17. McLean SG, Lipfert SW, van den Bogert AJ. Effect of gender and defensive opponent on the biomechanics of sidestep cutting. Med Sci Sports Exerc. 2004;36(6):1008-16.

18. Salci Y, Kentel BB, Heycan C, Akin S, Korkusuz F. Comparison of landing maneuvers between male and female college volleyball players. Clin Biomech. 2004;19(6):622-8.

19. Jacobs CA, Uhl T, Mattacola CG. Sex differences in eccentric hip-abductor strength and knee-joint kinematics when landing from a jump. J Sports Rehabil. 2005;14:346-55.

20. McLean SG, Huang X, Su A, van den Bogert AJ. Sagittal plane biomechanics cannot injure the ACL during sidestep cutting. Clin Biomech. 2004;19(8):828-38.

21. McLean SG, Su A, van den Bogert AJ. Development and validation of a $3 \mathrm{D}$ model to predict knee joint loading during dynamic movement. J Biomech Eng. 2003;31:864-74.

22. Fulkerson JP. Diagnosis and treatment of patients with patellofemoral pain. Am J Sports Med. 2002; $30(3): 447-56$.

23. Kirkendall DT, Garret WEJ. The anterior cruciate ligament enigma. Injury mechanisms and prevention. Clin Orthop Relat Res. 2000;(372):64-8.

24. Fung DT, Zhang LQ. Modeling of ACL impingement against the intercondylar notch. Clin Biomech. 2003; 18(10):933-41.

25. Hewett TE, Myer GD, Ford KR, Heidt RS, Coloismo AJ, McLean SG, et al. Biomechanical measures of neuromuscular control and valgus loading of the knee predict anterior cruciate ligament injury risk in female athletes. Am J Sports Med. 2005;33(4):492-501.

26. Ford KR, Myer GD, Hewett TE. Valgus knee motion during landing in high school female and male basketball players. Med Sci Sports Exerc. 2003;35(10):1745-50.

27. Russell KA, Palmieri RM, Zinder SM, Ingersoll CD. Sex differences in valgus knee angle during a single leg drop jump. J Athl Train. 2006;41(2):166-71. 
28. Hurd WJ, Chmielewski TL, Axe MJ, Davis I, SnyderMackler L. Differences in normal and perturbed walking kinematics between male and female athletes. Clin Biomech. 2004;19(5):465-72.

29. Ford KR, Myer GD, Smith RL, Vianello RM, Seiwert SL, Hewett TE. A comparison of dynamic coronal plane excursion between matched male and female athletes when performing single leg landings. Clin Biomech. 2006;21(1):33-40.

30. Ford KR, Myer GD, Toms HE, Hewett TE. Gender differences in the kinematics of unanticipated cutting in young athletes. Med Sci Sports Exerc. 2005; 37(1):124-9.

31. Zeller BL, McCrory JL, Kibler B, Uhl TL. Differences in kinematics and electromyographic activity between men and women during the single-legged squat. Am J Sports Med. 2003;31(3):449-56.

32. Willson JD, Ireland ML, Davis I. Core strength and lower extremity alignment during single leg squats. Med Sci Sports Exerc. 2006;38(5):945-52.

33. Claiborne TL, Armstrong CW, Gandhi V, Pincivero DM. Relationship between hip and knee strength and knee valgus during a single leg squat. J Appl Biomech. 2006;22(1):41-50.

34. Mandelbaum BR, Silvers HJ, Watanabe DS, Knarr JF, Thomas SD, Griffin LY, et al. Effectiveness of a neuromuscular and proprioceptive training program in preventing anterior cruciate ligament injuries in female athletes: 2-years follow-up. Am J Sports Med. 2005;33(7):1003-10.

35. Hewett TE, Stroupe AL, Nance TA, Noyes FR. Plyometric training in female athletes: decreased impact forces and increased hamstring torques. Am J Sports Med. 1996;24(6):765-73.
36. Noyes FR, Barber-Westin SD, Fleckenstein C, Walsh C, West J. The drop-jump screening test: difference in lower limb control by gender and effect of neuromuscular training in female athletes. Am J Sports Med. 2005;33(2):197-207.

37. Myer GD, Ford KR, Palumbo JP, Hewett TE. Neuromuscular training improves performance and lowerextremity biomechanics in female athletes. J Strength Cond Res. 2005;19(1):51-60.

38. Myer GD, Ford KR, McLean SG, Hewett TE. The effects of plyometric versus dynamic stabilization and balance training on lower extremity biomechanics. Strength Cond Res. 2006;20(2):345-53.

39. Pollard CD, Sigward SM, Ota S, Langford K, Powers CM. The influence of in-season injury prevention training on lower-extremity kinematics during landing in female soccer players. Clin J Sport Med. 2006; 16(3):223-7.

40. Herman DC, Weinhold PS, Guskiewicz KM, Garret WE, Yu B, Padua DA. The effects of the strength training on the lower extremities biomechanics of female recreational athletes during a stop-jump task. Am J Sports Med. 2008;36(4):733-40.

Recebido: $16 / 05 / 2010$

Received: 05/16/2010

Aprovado: $24 / 09 / 2010$

Approved: 09/24/2010 